\title{
The Role of Antidiuretic Hormone in Hyponatremia in Adrenal Insufficiency-Is the Guideline for the Diagnosis of Syndrome of Inappropriate Secretion of the Antidiuretic Hormone Appropriate?-
}

Key words: diuresis, glucocorticoid, Oleesky test

Syndrome of inappropriate secretion of the antidiuretic hormone (SIADH), was first described by Schwartz et al (1) in patients with bronchogenic carcinoma who were hyponatremic, but clinically euvolemic with normal renal and adrenal function in 1957. Long lists of disorders which can be associated with SIADH were proposed by many investigators (2-4), but all excluded adrenal insufficiency. Numerous criteria or guides for the diagnosis of SIADH $(3,4)$ emphasize normal adrenal function as an important diagnostic category. Clinical practitioners readily believe and follow these criteria and give the diagnosis of SIADH to their hyponatremic patients simply after confirming their patient's adrenal corticosteroid level to be normal. To begin with, hyponatremia is the most common disorder of body fluid and electrolyte balance encountered in clinical practice of medicine; the incidence ranges from 1 to $2.5 \%$ in chronically hospitalized patients (5). The problem is that hyponatremia is the most common laboratory finding in patients with acute and chronic adrenocortical insuffiency, either primary or secondary due to adrenocorticotropic hormone (ACTH) deficiency or tertially due to corticotropin-releasing hormone $(\mathrm{CRH})$ deficiency. The clinico-pathological findings of hyponatremia in some of the patients with adrenal insufficiency are similar to SIADH characterized by sodium loss without edema or dehydration, partly because of impaired water diuresis. Diuretic effects of glucocorticoids are well known for water dysuresis in adrenal insufficiency as has been demonstrated in the Oleesky test (6). In the absence of adrenal steroids, the kidneys are unable to excrete a water load and the defect is restored by glucocorticoids. The effect of glucocorticoid can be explained by improved renal hemodynamics and the effect on permeability of the renal tubule epithelium to water. In addition, a participation of antidiuretic hormone (ADH) must be taken into consideration as Shibata et al (7) described in this issue of Internal Medicine.

\section{See also p 426.}

The inhibitory effects of glucocorticoids on hypothalamic control of vasopressin release has been suggested for a long time in normal and adrenal deficient subjects based on clinical observations (8-10) or bioassay of ADH (11). After the establishment of a sensitive radioimmunoassay of $\mathrm{ADH}$, there came reports demonstrating a role of $\mathrm{ADH}$ in hyponatremic patients with hypoadrenalism due to hypopituitarism $(12,13)$. Serum arginine vasopressin (AVP) was inappropriately high in relation to low serum osmolality in these patients. Shibata et al (7) confirmed that the ADH secretion increased inappropriately to osmolality and the osmotic threshold of ADH secretion increased after glucocorticoid administration in their patient with adrenal insufficiency due to hypothalamic dysfunction who was initially diagnosed as SIADH in this issue of Internal Medicine.

Thus, it is evident that hyponatremia in adrenal insufficiency is partly caused by the inappropriate secretion of ADH. Vice versa, SIADH is caused by adrenal insufficiency. Partial adrenal insufficiency, which is defined as a normal basal plasma cortisol level with an impaired response to secretory stimuli, should not be overlooked in the 3 types of adrenal insufficiencies. Assessment of adrenocortical function using provocation test or a trial of glucocorticoids are needed in some of the patients with the slightest possibility of adrenal insufficiencies. Although the incidence or the exact mechanism of SIADH in adrenal deficient patients must be looked into further, it is recommended to delete the category of "normal adrenal function" from the criteria for the diagnosis of SIADH.

$$
\begin{array}{r}
\text { Reiko DEMURA, MD } \\
\text { The Department of Internal Medicine, } \\
\text { Institute of Clinical Endocrinology, } \\
\text { Tokyo Women's Medical University, } \\
\text { Tokyo 162-0054 }
\end{array}
$$

\section{References}

1) Schwartz WB, Bennett W, Curelop S, Bartter FC. A syndrome of renal sodium loss and hyponatremia probably resulting from inappropriate secretion of antidiuretic hormone. Am J Med 23: 529-542, 1957.

2) Bichet DG. The posterior pituitary in: The Pituitary, Melmed S, Ed. Blackwell Sci Inc, Boston, 1995: 299.

3) Robertson GL. The physiopathology of ADH secretion. in: Clinical Neuroendocrinology: A Pathophysiological Approach, Tolis et al, Eds. Raven Press, New York, 1979: 247-260. 
4) Saito T. SIADH and other hyponatremic disorders: diagnosis and therapeutic problems. Nippon Jinzo Gakkai Shi 38: 429-434, 1996.

5) Anderson RJ, Chung HM, Kluge R, Schrier RW. Hyponatremia: a prospective analysis of its epidemiology and the pathogenetic role of vasopressin. Ann Intern Med 102: 164-168, 1985.

6) Oleesky SA. Specific water diuresis test for adrenocortical insufficiency. Lancet 1: 769, 1953.

7) Shibata T, Oeda T, Saito Y. Severe hyponatremia caused by hypothalamic adrenal insufficiency. Intern Med 38: 426-432, 1999.

8) Dingman JP, Despointes RH. Adrenal steroid inhibition of vasopressin release from the neurohypophysis of normal subjects and patients with Addison's disease. J Clin Inv 39: 1851-1863, 1960.

9) Moses AM, Streeten DH. Differentiation of polyuric states by measurement of responses to changes in plasma osmolality induced by hyper- tonic saline infusions. Am J Med 42: 368-337, 1967.

10) Agus ZS, Goldberg M. Role of antidiuretic hormone in the abnormal water diuresis of anterior hypopituitarism in man. J Clin Inv 50: 1478-1489, 1971.

11) Kleeman CR, Czaczkes JW, Culter R. Mechanism of impaired water excretion in adrenal and pituitary insufficiency. IV. Antidiuretic hormone in primary and secondary adrenal insufficiency. J Clin Inv 43: 1641-1648, 1964.

12) Oelkers W. Hyponatremia and inappropriate secretion of vasopressin (antidiuretic hormone) in patients with hypopituitarism. New Engl J Med 321: 492-496, 1989.

13) Sakurai A, Yamada T, Hashizume $K$. A case of initially undiagnosed hypoadrenalism presenting inappropriate secretion of anti-diuretic hormone. Endocr J 42: 811-815, 1995. 